\title{
INVARIANCE GROUP PROPERTIES AND EXACT SOLUTIONS OF EQUATIONS DESCRIBING TIME-DEPENDENT FREE SURFACE FLOWS UNDER GRAVITY*
}

\author{
BY \\ P. L. SACHDEV (Indian Institute of Science, Bangalore) \\ AND \\ VARUGHESE PHILIP ( Regional Engineering College, Calicut)
}

\begin{abstract}
Summary. Using the method of infinitesimal transformations, a 6-parameter family of exact solutions describing nonlinear sheared flows with a free surface are found. These solutions are a hybrid between the earlier self-propagating simple wave solutions of Freeman, and decaying solutions of Sachdev. Simple wave solutions are also derived via the method of infinitesimal transformations. Incomplete beta functions seem to characterize these (nonlinear) sheared flows in the absence of critical levels.
\end{abstract}

1. Introduction. Nonlinear waterwave problems with a free surface present serious analytic difficulties in view of the rather awkward boundary conditions at the surface. Even in the shallow water approximation, exact solutions have been rare. Freeman [1] found a class of exact solutions of free surface flows under the assumption that the flow is a simple or traveling wave in the horizontal direction, the wave speed being given by $c \sim(g h)^{1 / 2}$, where $h=h(x, t)$ is the height of the free surface. Freeman's study was followed by Sachdev [2] who found exact time-dependent flows for which the free surface decayed as $t^{-2}$, in contrast to Freeman's (nondecaying) self-propagating flows in the horizontal direction. These (families of) solutions were found by heuristic and intuitive arguments without the use of systematic group analysis such as in the method of infinitesimal transformations. The solutions of both Freeman and Sachdev were expressed in terms of incomplete beta functions.

In the present paper, we undertake a more systematic approach to discovering new exact solutions of PDE's describing free surface flows under gravity by the method of infinitesimal transformations. Besides finding the new exact solutions, which we presently describe, the technique used here, though standard, is of interest in its own right for two reasons: (i) the system of nonlinear PDE's has three independent variables and therefore

\footnotetext{
* Received June 5, 1984.
} 
requires two-step reductions to nonlinear ODE's, (ii) the free surface boundary conditions are rather uncommon and their invariance requires a careful treatment. The consequence of this systematic (infinitesimal) group analysis is that a whole new class of 6-parameter family of solutions is found, which gives Sachdev's solution [2] as a special case. The form of the free surface is a hybrid between those of Freeman and Sachdev.

$$
h=\frac{k\left[x+A+\left(a_{0} / c\right)(t+B /(l+1))\right]^{2}}{(t+B)^{2}}
$$

in that it has a traveling wave form as well as explicit time decay. We also find another (singular) 5-parameter class of solutions besides the one just referred to. These solutions, in particular the singular ones, would be difficult to predict by intuitive arguments alone. This was also found to be the case in an earlier study, Sachdev and Reddy [3], wherein new gas-dynamic solutions describing flows headed by shocks were discovered, and the role of singular solutions (for some special relation among the parameters) was clearly brought out. The simple wave form of the shallow water equations was also subjected to infinitesimal transformations, but this did not lead to any new solutions besides those of Freeman [1]. However, an interesting feature of the present study is the method of solution of the nonlinear ODE's that result from self-similar form. Unlike the transformations used by Freeman and Sachdev, we exactly linearize the ODE's through a set of transformations into hypergeometric equations which have incomplete beta functions as their solutions. An important result that emerges from these studies is that all such (exact) free surface flows without critical levels (when they exist) are described by incomplete beta functions, a fact confirmed by another independent study of a related problem by Sachdev and Singh [4].

In Sec. 2, we give the basic shallow water equations and boundary conditions in the form suggested by Blythe et al. [5], using the so-called sigma variables, as well as the simple wave reduction of the equations [1]. Section 3 details the application of the method of infinitesimal transformations and the identification of the similarity variables. The new solutions are found in Sec. 4. Section 5 deals with simple wave equations and their solutions. The results and conclusions are contained in Sec. 6.

2. Basic equations. The unsteady two-dimensional inviscid flow equations in the "hydraulic approximation" are

$$
\begin{aligned}
u_{x}+v_{y} & =0, \\
u_{t}+u u_{x}+v u_{y}+g h_{x} & =0,
\end{aligned}
$$

where $x$ and $y$ are Cartesian coordinates measured along and perpendicular to the uniform horizontal bottom, $u$ and $v$ are the associated velocity components and $g$ is the acceleration due to gravity. The hydraulic approximation in the above equations replaces the pressure $p$ in (2.2) by the uniform gravitational pressure. This implies the shallow water approximation $H_{0} / L \ll 1$, where $H_{0}$ is a characteristic depth and $L$ a typical wavelength. The fluid depth $h(x, t)$ is to be determined such that the following boundary conditions are satisfied. 
At the bottom

$$
v=0 \quad \text { on } y=0 \text {. }
$$

On the free surface,

$$
v=h_{t}+u h_{x} \text { on } y=h .
$$

The condition that the pressure is constant on the free surface has been incorporated in the hydraulic approximation $p=p_{0}+g(h-y)$.

To simplify the boundary conditions, the independent and dependent variables are changed as follows:

$$
z=\frac{y}{h(x, t)}, \quad w=\frac{D z}{D t}=v-z\left(h_{t}+u h_{x}\right) .
$$

In terms of these variables, (2.1) and (2.2) become

$$
\begin{gathered}
h_{t}+u h_{x}+h\left(u_{x}+w_{z}\right)=0, \\
u_{t}+u u_{x}+w u_{z}+g h_{x}=0,
\end{gathered}
$$

while the boundary conditions (2.3) and (2.4) simplify to

$$
w=0 \quad \text { on } z=0,1 .
$$

For future reference, we also derive here the basic equations under the assumption of a simple wave flow, Freeman [1].

These flows are sought in the form

$$
\begin{aligned}
& u=u(x-c t, y), \\
& h=h(x-c t),
\end{aligned}
$$

where $c=c(h)$ only. With $\xi=x-c t$, Eqs. (2.1) and (2.2) may be written using the notation of Freeman [1] as

$$
\begin{array}{r}
\frac{\partial u}{\partial \xi}+\frac{\partial w}{\partial y}=0, \\
(u-c) \frac{\partial u}{\partial \xi}+w \frac{\partial u}{\partial y}+g \frac{d h}{d \xi}=0
\end{array}
$$

where $w=v(\partial \xi / \partial x)^{-1}$. Since $h=h(\xi)$, these equations may be written in terms of the independent variables $y$ and $h$,

$$
\begin{array}{r}
\frac{\partial u}{\partial h}+\frac{\partial w_{1}}{\partial y}=0, \\
(u-c) \frac{\partial u}{\partial h}+w_{1} \frac{\partial u}{\partial y}+g=0,
\end{array}
$$

with boundary conditions

$$
\begin{aligned}
& w_{1}=0 \text { on } y=0, \\
& w_{1}=u-c, \quad(u-c) \frac{\partial u}{\partial h}+g=0 \quad \text { on } y=h,
\end{aligned}
$$


where

$$
w_{1}=w \frac{d \xi}{d h}=v \frac{(d \xi / d h)}{(\partial \xi / \partial x)}=\frac{v}{\left(\frac{\partial h}{\partial x}\right)}
$$

3. Invariance group and similarity variables. To identify the similarity form, we use the theory of infinitesimal transformations given originally by Ovsjannikov [6] and expounded more clearly by Bluman and Cole [7]. We employ an abbreviated form the same as in the paper of Logan and Perez [8]. We seek a one-parameter infinitesimal group of transformations which takes the $(t, x, z, u, w)$ space into itself and under which the differential equations (2.6)-(2.7) are invariant:

$$
\begin{aligned}
& t^{*}=t+\in T, \quad x^{*}=x+\in X, \quad z^{*}=z+\in Z, \\
& u^{*}=u+\in U, \quad w^{*}=w+\in W,
\end{aligned}
$$

where the generators $T, X, Z, U$, and $W$ are functions of $t, x, z, u$, and $w$.

We introduce the following notations for convenience. Let $x^{1}=t, x^{2}=x, x^{3}=z$. $u^{1}=u, u^{2}=w$ and define the derivatives

$$
p_{j}^{i}=\partial u^{i} / \partial x^{j}, \quad i=1,2 ; j=1,2,3 .
$$

It can be seen that the invariance of the differential equations as formulated by Bluman and Cole [7] is equivalent to the following definition of invariance [8]: a system of differential equations

$$
H_{n}\left(x^{j}, u^{k}, p_{j}^{i}\right)=0, \quad n=1,2,
$$

is said to be constantly conformally invariant under the infinitesimal group (3.1) if there exist constants $\alpha_{n i}(n, i=1,2)$ such that

$$
L H_{n}=\alpha_{n i} H_{i}, \quad n=1,2,
$$

for all smooth surfaces $u^{i}=u^{i}\left(x^{j}\right)$, where $L$ is the Lie derivative in the direction of the extended vector field,

$$
L=A=\xi_{x}^{j} \frac{\partial}{\partial x^{j}}+\xi_{u}^{i} \frac{\partial}{\partial u^{i}}+\xi_{p j}^{i} \frac{\partial}{\partial p_{j}^{i}}
$$

and where

$$
\begin{array}{ll}
\xi_{x}^{1}=T, & \xi_{x}^{2}=X, \quad \xi_{x}^{3}=Z, \\
\xi_{u}^{1}=U, & \xi_{u}^{2}=W .
\end{array}
$$

Further,

$$
\xi_{p j}^{i}=\frac{\partial \xi_{u}^{i}}{\partial x^{j}}+\frac{\partial \xi_{u}^{i}}{\partial u^{k}} p_{j}^{k}-\frac{\partial \xi_{x}^{k}}{\partial x_{j}^{j}} p_{k}^{i}-\frac{\partial \xi_{x}^{k}}{\partial u^{m}} p_{k}^{i} p_{j}^{m}
$$


are the generators of the derivative transformations. Writing out (3.2), we have

$$
\frac{\partial H_{n}}{\partial x^{j}} \xi_{x}^{j}+\frac{\partial H_{n}}{\partial u^{i}} \xi_{u}^{i}+\frac{\partial H_{n}}{\partial p_{j}^{i}} \xi_{p j}^{i}=\alpha_{n i} H_{i}, \quad n=1,2 .
$$

Substituting $\xi_{p j}^{i}$ from (3.3) into (3.4) we get polynomial (equations) in $p^{j}$. If these solutions are to hold for arbitrary $p_{j}^{i}$, the coefficients of $p_{j}^{i}$ must vanish. We thus obtain a system of linear PDE's in the generators $T, X, Z, U$, and $W$. This system of equations, of ten referred to as the determining system, is solved to find the group (3.1).

The invariance of (2.6) gives

$$
\begin{aligned}
& T=T(t, x, z), \quad X=X(t, x, z), \quad Z=Z(t, x, z), \\
& h\left(U_{u}-X_{v}\right)+X h_{x}+T h_{t}=\alpha_{11} h+\alpha_{12} u, \\
& h\left(W_{u}-Z_{v}\right)=\alpha_{12} w \text {, } \\
& -h T_{\mathrm{v}}=\alpha_{12} \text {, } \\
& h\left(U_{n}-X_{z}\right)=0 \text {, } \\
& h\left(W_{\text {n }}-Z_{z}\right)+\left(X h_{x}+T h_{t}\right)=\alpha_{11} h, \\
& h T_{z}=0 \text {, } \\
& X h_{x}+T h_{t \prime}+u\left(X h_{x x}+T h_{x t}\right)+U h_{x}+h U_{x}+h W_{z} \\
& =\alpha_{11}\left(h_{t}+u h_{x}\right)+\alpha_{12} g h_{x} .
\end{aligned}
$$

Invariance of (2.7) yields

$$
\begin{aligned}
U+u\left(U_{u}-X_{x}\right)-w X_{z}-X_{t} & =\alpha_{21} h+\alpha_{22} u, \\
W+w\left(U_{u}-Z_{z}\right)-u Z_{x}-Z_{t} & =\alpha_{22} w, \\
U_{u}-T_{t}-u T_{x}-w T_{z} & =\alpha_{22}, \\
u W_{u} & =0, \\
w U_{x} & =\alpha_{21} h, \\
U_{x} & =0, \\
U_{t}+u U_{x}+w U_{z}+g\left(X h_{x x}+T h_{x t}\right) & =\alpha_{21}\left(h_{t}+u h_{x}\right)+\alpha_{22} g h_{x} .
\end{aligned}
$$

We now solve the determining differential equations above for the transformation group. Differentiating (3.7) with respect to $w$ and (3.10) with respect to $u$ we get $\alpha_{12}=W_{u w^{\prime}}=0$. Equations (3.18) and (3.17) imply $\alpha_{21}=0(h \neq 0)$. Differentiating (3.13) with respect to $w$ and using (3.18) and (3.5) we get $X=X(x, t)$. Equations (3.11), (3.8), and (3.5) now imply that $T=T(t)$. Further, Eq. (3.6) gives $U_{u u}=0$. Equations (3.10), (3.12), and (3.7) give $W_{w w^{\prime}}=W_{z w^{\prime}}=W_{u u}=0=W_{u u^{*}}$. These equations yield

$$
W=k(x, t) w+l_{1}(x, z, t) u+m(x, z, t),
$$

where $k, l_{1}$, and $m$ are arbitrary functions. Equation (3.19) differentiated with respect to $w$ gives $U_{z}=0$, which implies that $U=U(t, x, u)$. Equation (3.15) now gives

$$
U=\left[\alpha_{22}+T^{\prime}(t)\right] u+f(x, t),
$$


where $f$ is an arbitrary function of $x$ and $t$. Differentiating (3.13) with respect to $u$ and using (3.21) we obtain

$$
X=\left[\alpha_{22}+2 T^{\prime}(t)\right] x+\beta(t),
$$

where $\beta$ is an arbitrary function of $t$. The equations obtained by differentiating (3.13) with respect to $x$ and (3.19) with respect to $u$ and using (3.21) and (3.22) give $f=f(t)$ and

$$
T=a t+b,
$$

where $a$ and $b$ are arbitrary constants. Differentiating (3.14) with respect to $w$ and using Eqs. (3.20), (3.21), and (3.23), we get $Z=[k(x, t)+a] z+\gamma(x, t)$, where $\gamma$ is an arbitrary function of $x$ and $t$. Invariance of the boundary $z=0$ implies $z^{*}=z+\in Z=0$, when $z=0$; this implies $Z=0$, when $z=0 \Rightarrow \gamma(x, t)=0$. Invariance of the other boundary $z=1$ implies that $z^{*}=z+\in Z=1$, when $z=1 \Rightarrow Z=0$, when $z=1 \Rightarrow k(x, t)=-a$. Thus we have $Z=0$. Equations (3.7) and (3.20) now give $l_{1}=0$, and (3.14) gives $m=0$. Eq. (3.13) provides that $f(t)=\beta^{\prime}(t)$. Thus the generators of the invariance group for the equations $(2.6)-(2.8)$ are

$$
\begin{aligned}
& T=a t+b, X=(c+a) x+\beta(t), Z=0, \\
& U=c u+\beta^{\prime}(t), W=-a w,
\end{aligned}
$$

where $a, b, c=\alpha_{22}+a$ are arbitrary constants and $\beta(t)$ is an arbitrary function of $t$.

Using (3.24) in (3.6) we get

$$
(a t+b) h_{t}+[(c+a) x+\beta(t)] h_{x}-\left(\alpha_{11}+a\right) h=0 .
$$

Differentiating (3.25) with respect to $x$ and combining it with the equation obtained by substituting (3.24) in (3.19), we have

$$
\left(2 c-a-\alpha_{11}\right) h_{x}=\frac{\beta^{\prime \prime}(t)}{g} .
$$

From (3.26) we get the following alternatives:

(i) $2 c=a+\alpha_{11}$, and therefore,

$$
\beta(t)=a_{0} t+a_{1},
$$

where $a_{0}$ and $a_{1}$ are arbitrary constants.

(ii)

$$
h=\frac{\beta^{\prime \prime}(t)}{g\left(2 c-a-\alpha_{11}\right)} x+\beta_{1}(t),
$$

where $\beta_{1}(t)$ is an arbitrary funtion of $t$, but this does not give any new solution.

The invariant surface conditions [7] for $u$ and $w$ are, respectively,

$$
\begin{gathered}
T \frac{\partial u}{\partial t}+X \frac{\partial u}{\partial x}+Z \frac{\partial u}{\partial z}=U, \\
T \frac{\partial w}{\partial t}+X \frac{\partial w}{\partial x}+Z \frac{\partial w}{\partial z}=W .
\end{gathered}
$$

Case (i), $c / a \neq-1$. 
The characteristic system of (3.28) is

$$
\frac{d t}{a t+b}=\frac{d x}{(c+a) x+a_{0} t+a_{1}}=\frac{d z}{0}=\frac{d u}{c u+a_{0}} .
$$

Integration of the first two equations gives the similarity variables:

$$
\begin{aligned}
\xi & =\left[x+A+\frac{a_{0}}{c}\left(t+\frac{B}{l+1}\right)\right] \frac{1}{(t+B)^{l+1}}, \\
\text { where } l & =c / a \neq-1, A=a_{1} /(c+a), \text { and } B=b / a(a \neq 0), \\
\eta & =z \text {. }
\end{aligned}
$$

Integration of the third equation gives the similarity form

$$
u=(t+B)^{\prime} F(\xi, \eta)-a_{0} / c,
$$

where $F(\xi, \eta)$ is an arbitrary function of $\xi$ and $\eta$. In a similar manner, Eq. (3.29) gives

$$
w=(t+B)^{-1} G(\xi, \eta),
$$

where $G(\xi, \eta)$ is another arbitrary function of $\xi$ and $\eta$. The characteristic system of (3.25), with $\beta(t)$ given by (3.27) and $2 c=a+\alpha_{11}$, is

$$
\frac{d t}{a t+b}=\frac{d x}{(c+a) x+a_{0} t+a_{1}}=\frac{d h}{2 c h},
$$

from which we get

$$
h=(t+B)^{2 l} s(\xi),
$$

where $s$ is an arbitrary function of $\xi$.

By substituting the self-similar (in $\xi$ and $\eta$ ) forms of the solutions (3.33), (3.34) in Eqs. (2.6) and (2.7) we obtain a system of PDE's with two independent variables, $\xi$ and $\eta$ :

$$
\begin{gathered}
F_{\xi}+G_{\eta}+[F-(l+1) \xi]\left(s_{\xi} / s\right)+2 l=0, \\
{[F-(l+1) \xi] F_{\xi}+G F_{\eta}+l F+g s_{\xi}=0 .}
\end{gathered}
$$

The method of infinitesimal transformation group is again applied to the system of PDE's (3.26) and (3.27) to reduce it to a system of ODE's. For convenience of further analysis we rename $\xi, \eta, F$, and $G$ as $x, z, u$, and $w$ respectively. The PDE's (3.36) and (3.37) can now be written as

$$
\begin{gathered}
u_{x}+w_{z}+[u-(l+1) x]\left(s_{x} / s\right)+2 l=0, \\
{[u-(l+1) x] u_{x}+w u_{z}+l u+g s_{x}=0 .}
\end{gathered}
$$

We again seek a one-parameter infinitesimal group of transformations

$$
\begin{aligned}
& x^{*}=x+\in X, \quad z^{*}=z+\in Z, \quad u^{*}=u+\in U, \\
& w^{*}=w+\in W,
\end{aligned}
$$

under which the system (3.38)-(3.39) is invariant. Here the generators $X, Z, U$, and $W$ are functions of $x, z, u$, and $w$. 
Invariance of (3.38) gives

$$
\begin{aligned}
& X=X(x, z), \quad Z=Z(x, z), \\
& U_{u}-X_{x}=\beta_{11}+\beta_{12}[u-(l+1) x], \\
& W_{u}-Z_{x}=\beta_{12} w, \\
& U_{w^{\prime}}-X_{z}=0 \\
& W_{w^{\prime}}-Z_{z}=\beta_{11}, \\
& U_{x}+W_{z}+[u-(l+1) x] X\left(s_{x} / s\right)_{x}+[U-(l+1) X]\left(s_{x} / s\right) \\
&= \beta_{11}[u-(l+1) x]\left(s_{x} / s\right)+2 l \beta_{11}+\beta_{2}\left(l u+g h_{x}\right) .
\end{aligned}
$$

Invariance of (3.39) yields

$$
\begin{gathered}
U-(l+1) X+[u-(l+1) x]\left(U_{u}-X_{x}\right)-w X_{z}=\beta_{21}+\beta_{22}[u-(l+1) x] \\
W+w\left(U_{u}-Z_{z}\right)-[u-(l+1) x] Z_{x}=\beta_{22} w \\
{[u-(l+1) x] U_{u^{\prime}}=0} \\
w U_{u}=\beta_{21} \\
{[u-(l+1) x] U_{x}+U_{z}+l U+g X_{s_{1}}} \\
=\beta_{21}[u-(l+1) x]\left(s_{x} / s\right)+2 l \beta_{21}+\beta_{22}\left(l u+g s_{x}\right)
\end{gathered}
$$

We now solve the determining differential Eqs. (3.41)-(3.51) for the transformation group (3.40). Equations (3.43) and (3.45) imply $W_{u \cdot u}=\beta_{12}=0$. Equations (3.49) and (3.50) give $U_{u}=0, u \neq(l+1) x, \beta_{21}=0$. Now (3.42) differentiated with respect to $u$ yields $U_{u u}=0$. Differentiating (3.43) with respect to $u$ and (3.45) and (3.46) with respect to $w$, we get $W_{u u}=W_{u \cdot w^{\prime}}=W_{z w^{\prime}}=0$. These equations together with $W_{u w}=0$ imply

$$
W=m(x) w+p(x, z) u+q(x, z),
$$

where $m, p, q$ are arbitrary functions of the indicated arguments. Using (3.52) in (3.45) we get $Z=\left[m(x)-\beta_{11}\right] z+f_{1}(x)$ where $f_{1}(x)$ is an arbitrary function of $x$. Invariance of $z=0$ implies $f_{1}=0$ and invariance of $z=1$ implies $m(x)=\beta_{11}$, so that

$$
Z=0 \text {. }
$$

Equations (3.43) and (3.53) give $W_{u}=0$ which, by the use of (3.52), implies $p=0$. Equation (3.51) differentiated with respect to $w$ gives $U_{z}=0$ so that $U=U(x, u)$, and (3.47) differentiated with respect to $w$ yields $X_{z}=0$ so that $X=X(x)$. Differentiating (3.47) with respect to $w$ and using (3.42) we get $2 U_{u}=\beta_{22}+X^{\prime}(x)=2\left[\beta_{11}+X^{\prime}(x)\right]$, from which we get

$$
X=a_{1} x+b_{1},
$$

where $a_{1}\left(=\beta_{22}-2 \beta_{11}\right)$ and $b_{1}$ are arbitrary constants. Therefore,

$$
U=\left(\beta_{11}+a_{1}\right) u+f_{2}(x),
$$


where $f_{2}$ is an arbitrary function of $x$. Equations (3.52), (3.53), (3.55), and (3.48) now give $q=0$, so that

$$
W=\beta_{11} w .
$$

.Equation (3.47) now gives $f_{2}(x)=(l+1)\left(b_{1}-\beta_{11} x\right)$ and so

$$
U=\left(\beta_{11}+a_{1}\right) u+(l+1)\left(b_{1}-\beta_{11} x\right) \text {. }
$$

Substituting (3.54), (3.56), and (3.57) in (3.46) and differentiating the result obtained with respect to $u$, we get

$$
\left(a_{1} x+b_{1}\right)\left(s_{x} / s\right)_{x}=-a_{1}\left(s_{x} / s\right)
$$

from which we get

$$
s(x)=k_{2}\left(a_{1} x+b_{1}\right)^{k_{1} / a_{1}},
$$

where $k_{1}$ and $k_{2}$ are arbitrary constants. Substituting (3.58) in (3.51) and equating to zero the coefficient of $u$ and the terms independent of $u$, respectively, we get $\beta_{11}=0$, provided $l \neq-\frac{1}{2}$, and

$$
l(l+1) b_{1}=g k_{1} k_{2}\left(a_{1} x+b_{1}\right)^{k_{1} / a_{1}-1}\left(2 a_{1}-k_{1}\right) .
$$

The only meaningful relation arising from (3.59) is $b_{1}=0, k_{1}=2 a_{1}$. Therefore, the infinitesimal generators, in terms of the original variables, $\xi, \eta, F$, and $G$ are

$$
\begin{aligned}
X & =a_{1} \xi, \quad Z=0, \quad U=a_{1} F, \quad W=0, \\
h & =(t+B)^{2 l} s(\xi)=(t+B)^{2 l} k_{2}\left(a_{1} \xi\right)^{2}=k(t+B)^{2 l} \xi^{2}, \\
& =\frac{k\left[x+A+\left(a_{0} / c\right)(t+B /(l+1))\right]^{2}}{(t+B)^{2}},
\end{aligned}
$$

where $k=k_{2} a_{1}^{2}$. Equations (3.30) and (3.61) show that there are six independent parameters, $a, b, c, a_{0}, a_{1}$, and $k$ characterizing these self-similar solutions. The invariant surface conditions for $F$ and $G$ are

$$
\begin{aligned}
& X \frac{\partial F}{\partial \xi}+Z \frac{\partial F}{\partial \eta}=U, \\
& X \frac{\partial G}{\partial \xi}+Z \frac{\partial G}{\partial \eta}=W .
\end{aligned}
$$

The characteristic system for (3.62) is

$$
\frac{d \xi}{a_{1} \xi}=\frac{d \eta}{0}=\frac{d F}{a_{1} F} .
$$

One first integral gives the similarity variable $\eta$. The second integral is

$$
F=\xi P(\eta),
$$

where $P$ is an arbitrary function of $\eta$. In a similar manner, (3.63) gives

$$
G=Q(\eta),
$$

where $Q$ is an arbitrary function of $\eta$. 
4. Time-dependent solutions. Substituting the self-similar forms (3.64) and (3.65) in (3.36) and (3.37) and using (3.61), we get the following system of ODE's wherein prime denotes differentiation with respect to $\eta$ :

$$
\begin{aligned}
3 P+Q^{\prime}-2 & =0, \\
Q P^{\prime}-P+P^{2}+2 g k & =0 .
\end{aligned}
$$

Denoting $g k$ by $\left(4 \alpha^{2}\right)^{-1}$ in (4.2), we have

$$
Q=\frac{P-P^{2}-1 / 2 \alpha^{2}}{P^{\prime}} .
$$

Substituting (4.3) in (4.1) we get

$$
\left(P^{2}-P+1 / 2 \alpha^{2}\right) P^{\prime \prime}+(P-1) P^{\prime 2}=0 .
$$

We write $P=U / 2 \alpha$. (U, here, is not the generator defined earlier.) Equation (4.4) now becomes

$$
\left(U^{2}-2 \alpha U+2\right) U^{\prime \prime}+(U-2 \alpha) U^{\prime 2}=0 .
$$

By interchanging the independent and dependent variables $\eta$ and $U,(4.5)$ transforms to

$$
\left(U^{2}-2 \alpha U+2\right) \frac{d^{2} \eta}{d U^{2}}+(2 \alpha-U) \frac{d \eta}{d U}=0,
$$

or

$$
\left(U-\lambda_{1}\right)\left(U-\lambda_{2}\right) \frac{d^{2} \eta}{d U^{2}}+(2 \alpha-U) \frac{d \eta}{d U}=0,
$$

where $\lambda_{1}$ and $\lambda_{2}$ are the roots of

$$
\lambda^{2}-2 \alpha \lambda+2=0,
$$

assumed to be real. Therefore $\alpha^{2}>2$. Also, $F \neq(l+1) \xi$. This is the condition for the absence of critical levels in the flow.

Putting $\tau=\left(U-\lambda_{1}\right) /\left(\lambda_{2}-\lambda_{1}\right)$ in Eq. (4.7) we have

$$
\tau(1-\tau) \frac{d^{2} \eta}{d \tau^{2}}-\left(\frac{2 \alpha-\lambda_{1}}{\lambda_{2}-\lambda_{1}}-\tau\right) \frac{d \eta}{d \tau}=0 .
$$

In view of the relations $2 \alpha=\lambda_{1}+\lambda_{2}=\lambda_{1}+2 / \lambda_{1}$ arising from (4.8), the above equation may be written as

$$
\tau(1-\tau) \frac{d^{2} \eta}{d \tau^{2}}+\left(\frac{2}{\lambda_{1}^{2}-2}+\tau\right) \frac{d \eta}{d \tau}=0,
$$

which is a hypergeometric equation having the solution, Luke [9],

$$
\begin{aligned}
\eta= & A_{1}+B_{1}\left(\frac{U-\lambda_{1}}{2 \lambda_{1}^{-1}-\lambda_{1}}\right)^{\left.\left(4-\lambda_{\mathrm{i}}^{2}\right) / 2-\lambda_{\mathrm{i}}^{2}\right)} \\
& \cdot 2 F_{1}\left(\frac{4-\lambda_{1}^{2}}{2-\lambda_{1}^{2}}, \frac{\lambda_{1}^{2}}{2-\lambda_{1}^{2}}, \frac{6-2 \lambda_{1}^{2}}{2-\lambda_{1}^{2}} ; \frac{U-\lambda_{1}}{2 \lambda_{1}^{-1}-\lambda_{1}}\right),
\end{aligned}
$$


where $A_{1}$ and $B_{1}$ are arbitrary constants. Using the relation

$$
\beta(p, q, x)=\frac{x^{p}}{p} 2 F_{1}\left(\begin{array}{rr}
p, & 1-q \\
p+1 ; & x
\end{array}\right)
$$

Luke [9]. (4.10) may be rewritten as

$$
\eta=A_{1}+B_{2} \beta\left(\frac{4-\lambda_{1}^{2}}{2-\lambda_{1}^{2}}, \frac{2-2 \lambda_{1}^{2}}{2-\lambda_{1}^{2}}, \frac{U-\lambda_{1}}{2 \lambda_{1}^{-1}-\lambda_{1}}\right)
$$

where $B_{2}$ is an arbitrary constant. From (4.3) we get

$$
Q=\frac{\left(U^{2}-2 \alpha U+2\right)}{2 \alpha U^{\prime}}=-\frac{\left(U-\lambda_{1}\right)\left(U-\lambda_{2}\right)}{2 \alpha U^{\prime}}
$$

so that the boundary conditions become $\eta=0, U=\lambda_{1}$ and $\eta=1, U=\lambda_{2}=2 / \lambda_{1}$. These require $A_{1}=0$ and

$$
B_{2}^{-1}=\beta\left(\frac{4-\lambda_{1}^{2}}{2-\lambda^{2}}, \frac{2-2 \lambda_{1}^{2}}{2-\lambda_{1}^{2}}, 1\right)
$$

Finally we get the solution

$$
\eta=\beta\left(\frac{4-\lambda_{1}^{2}}{2-\lambda_{1}^{2}}, \frac{2-2 \lambda_{1}^{2}}{2-\lambda_{1}^{2}}, \frac{U-\lambda_{1}}{2 \lambda_{1}^{-1}-\lambda_{1}}\right) / \beta\left(\frac{4-\lambda_{1}^{2}}{2-\lambda_{1}^{2}}, \frac{2-2 \lambda_{1}^{2}}{2-\lambda_{1}^{2}}, 1\right) .
$$

If we take $a_{0}=0, B=0, A=0$ and $k=1$, we get Sachdev's solution [2] (after correcting a minor misprint in Eqs. (3.17)-(3.18) of [2]).

Case (ii). $c / a=-1$. Similarity form (3.33) was derived on the assumption $c / a \neq-1$. Now we consider the special singular case $c / a=-1$. The characteristic system for (3.28) in this case is given by

$$
\frac{d t}{a t+b}=\frac{d x}{a_{0} t+a_{1}}=\frac{d z}{0}=\frac{d u}{-a u+a_{0}} .
$$

Integrating the first two of these equations we have

$$
\begin{aligned}
& \xi=x-A_{0} t-\left(A_{1}-A_{0} B\right) \ln (t+B), \\
& \eta=z,
\end{aligned}
$$

where $A_{0}=a_{0} / a, A_{1}=a_{1} / a$ and $B=b / a$. The last equation integrates to give

$$
u=A_{0}+(t+B)^{-1} F(\xi, \eta),
$$

where $F$ is an arbitrary function of $\xi$ and $\eta$. From the characteristic system for (3.29) we similarly get

$$
w=(t+B)^{-1} G(\xi, \eta),
$$

where $G$ is another arbitrary function of $\xi$ and $\eta$. The characteristic system for (3.25) now is

$$
\frac{d t}{a t+b}=\frac{d x}{a_{0} t+a_{1}}=\frac{d h}{-2 a h} .
$$


Its general solution is

$$
h=(t+B)^{-2} s(\xi)
$$

where $s$ is an arbitrary function of $\xi$. Substituting (4.13)-(4.14) in (2.6) and (2.7), we obtain

$$
\begin{aligned}
& F_{\xi}+G_{\eta}+\left(F-A_{2}\right)\left(s_{\xi} / s\right)-2=0, \\
& \left(F-A_{2}\right) F_{\xi}+G F_{\eta}-F+g s_{\xi}=0,
\end{aligned}
$$

where $A_{2}=A_{1}-A_{0} B$. Rewriting $\xi, \eta, F$, and $G$ as $x, z, u$, and $w$ for the sake of convenience, (4.18) and (4.19) may be rewritten as

$$
\begin{aligned}
& u_{x}+w_{z}+\left(u-A_{2}\right)\left(s_{x} / s\right)-2=0, \\
& \left(u-A_{2}\right) u_{x}+w u_{z}-u+g s_{x}=0 .
\end{aligned}
$$

The determining differential equations for the transformation group (3.40) which leave (4.20) and (4.21) invariant can be obtained from (3.41)-(3.51) simply by replacing the term $(l+1) x$ by $A_{2}$ and $l$ by -1 . Proceeding exactly as above we get $A_{2}=0$. Therefore,

$$
\xi=x-A_{0} t .
$$

The generators are

$$
X=a_{1} \xi+b_{1}, \quad Z=0, \quad U=a_{1} F, \quad W=0 .
$$

Also,

$$
\begin{aligned}
h & =\frac{k_{2}\left(a_{1} \xi+b_{1}\right)^{2}}{(t+B)^{2}} \\
& =\frac{k_{2} a_{1}^{2}\left(x+B_{1}-A_{0} t\right)^{2}}{(t+B)^{2}}=\frac{k\left(x+B_{1}-A_{0} t\right)^{2}}{(t+B)^{2}},
\end{aligned}
$$

where $B_{1}=b_{1} / a_{1}$. Equations (4.13) and (4.24) show that there are 5 independent parameters $a_{0}, a_{1}, a, b$, and $k$ characterizing these solutions. The characteristic system for the invariant surface condition (3.62) is

$$
\frac{d \xi}{a_{1} \xi+b_{1}}=\frac{d \eta}{0}=\frac{d F}{a_{1} F} .
$$

Integration of these equations gives $\eta$ as a similarity variable and

$$
F=\left(\xi+B_{1}\right) P(\eta) \text {. }
$$

Similarly, from (3.63) we get

$$
G=Q(\eta)
$$

$P(\eta)$ and $Q(\eta)$ are arbitrary functions of $\eta$. Equations (4.18) and (4.19) now become

$$
\begin{aligned}
& 3 P+Q^{\prime}-2=0, \\
& P^{2}+Q P^{\prime}-P+2 g k=0,
\end{aligned}
$$

which are the same as (4.1) and (4.2). Now proceeding as in the case of Eqs. (4.1) and (4.2), we get another solution in the form (4.12). 
5. Simple wave solutions. We now assume a (nonlinear) simple wave form of the solution in the horizontal $(x)$ direction and seek the infinitesimal group of transformations which leaves the simple wave equations invariant. That is, we transform the system (2.9)-(2.10) according to

$$
h^{*}=h+\in H, \quad y^{*}=y+\in Y, \quad u^{*}=u+\in U, \quad w^{*}=w+\in W,
$$

where the generators $H, Y, U$, and $W$ are functions of $h, y, u$, and $w$. For convenience, we use here $w$ instead of $w_{1}$.

Invariance of (2.9) gives

$$
\begin{aligned}
& H=H(h, y), \quad Y=Y(h, y), \\
& U_{u}-H_{h}=\alpha_{11}+\alpha_{12}(u-c), \\
& W_{u}-Y_{h}=\alpha_{12} w, \\
& U_{w}-H_{y}=0 \\
& W_{w^{\prime}}-Y_{y}=\alpha_{11}, \\
& U_{h}-W_{y}=\alpha_{12} g .
\end{aligned}
$$

Invariance of (2.10) requires

$$
\begin{aligned}
& U-H c_{h}+(u-c)\left(U_{u}-H_{h}\right)-w H_{y}=\alpha_{21}+\alpha_{22}(u-c), \\
& W-(u-c) Y_{h}+w\left(U_{u}-Y_{y}\right)=\alpha_{22} w, \\
& (u-c) U_{w}=0, \\
& w U_{w}=\alpha_{21}, \\
& (u-c) U_{h}+w U_{y}=\alpha_{22} g .
\end{aligned}
$$

Assuming $u \neq c$, (5.4) and (5.6) yield $W_{u w}=\alpha_{12}=0$, while (5.10) and (5.11) give $U_{n^{\prime}}=0=\alpha_{21}$. Equation (5.5) now implies $H=H(h)$. Differentiation of (5.12) with respect to $w$ gives $U_{y}=0$ and (5.12) then implies $U_{h h}=0$. From (5.3), (5.4), (5.6), and (5.7) we get $U_{u u}=W_{u u}=W_{w w}=W_{y y}=0$. Differentiating (5.9) with respect to $w$ we have $W_{w}+U_{u}-Y_{y}=\alpha_{22}$. Adding (5.3) and (5.6) we get $W_{w}+U_{u}-Y_{y}-H_{h}=2 \alpha_{11}$. These two equations give $H_{h}=\alpha_{22}-2 \alpha_{11}=a$ and hence

$$
H=a h+b,
$$

where $a$ and $b$ are arbitrary constants. Using (5.13) in (5.3) we get

$$
U=\left(\alpha_{11}+a\right) u+f_{1} h+f,
$$

where $f_{1}$ and $f$ are arbitrary constants and use is made of the equation $U_{h h}=0$. Equation (5.7) gives $W_{y}=-U_{h}=-f_{1}$ so that

$$
W=-f_{1} y+l_{1}(h) w+k_{1}(h) u+m_{1}(h),
$$

where $l_{1}, k_{1}, m_{1}$ are arbitrary functions of $h$. Eq. (5.6) yields $Y=\left[l(h)-\alpha_{11}\right] y+m(h)$, where $m$ is an arbitrary function of $h$. Invariance of $y=0$ implies $m(h)=0$, and so $Y=\left[l(h)-\alpha_{11}\right] y$. Using this equation and (5.15) in (5.4), we get $k_{1}(h)-l^{\prime}(h) y=0$ which implies $k_{1}(h)=0$ and $l(h)=l$, a constant, so that

$$
Y=\left(l-\alpha_{11}\right) y \text {. }
$$


Now (5.9) gives $-f_{1} y+m_{1}(h)=0$ from which we have $f_{1}=0$ and $m_{1}=0$ so that $U=\left(\alpha_{11}+a\right) u+f, W=l w$. Using these in (5.12) we get $\alpha_{22}=0$ and therefore $\alpha_{11}=$ $-a / 2$. Invariance of $y=h$ implies $Y=H$ when $y=h \Rightarrow(l+a / 2) h=a h+b$ which gives $l=a / 2, b=0$. Thus, the generators of the infinitesimal transformation group (5.1) are

$$
H=a h, \quad Y=a y, \quad U=a u / 2+f, \quad a w / 2 .
$$

Using (5.17) in (5.8) we get

$$
\frac{d c}{d h}-\frac{c}{2 h}=\frac{f}{a h}
$$

the solution of which is

$$
c=K h^{1 / 2}-\frac{2 f}{a} .
$$

The invariant surface conditions [7] for $u$ and $w$ are

$$
\begin{gathered}
H \frac{\partial u}{\partial h}+Y \frac{\partial u}{\partial y}=U \\
H \frac{\partial w}{\partial h}+Y \frac{\partial w}{\partial y}=W
\end{gathered}
$$

The characteristic system for (5.19) is

$$
\frac{d h}{a h}=\frac{d y}{a y}=\frac{d u}{a u / 2+f} .
$$

A first integral gives the similarity variable

$$
\eta=y / h .
$$

A second integral gives

$$
u=y^{1 / 2} P(\eta)-\frac{2 f}{a},
$$

where $P$ is an arbitrary function of $\eta$. Similarly from the characteristic system of (5.20) we get

$$
w=y^{1 / 2} Q(\eta),
$$

where $Q$ is an arbitrary function of $\eta$. Using (5.22) and (5.23), Eq. (2.9) transforms to

$$
2 \eta^{2} P^{\prime}-2 \eta Q^{\prime}-Q=0
$$

where prime denotes differentiation with respect to $\eta$. Equation (2.10) transforms to

$$
\left(\frac{1}{2} P+\eta P^{\prime}\right) Q-\eta^{2} P P^{\prime}+2 \alpha g^{1 / 2} \eta^{3 / 2} P^{\prime}+g=0,
$$

where we have chosen the constant $K=2 \mathrm{\alpha g}^{1 / 2}$, for convenience. The above equation may be written as

$$
Q=\frac{\eta^{2} P P^{\prime}-2 \alpha g^{1 / 2} \eta^{3 / 2} P^{\prime}-g}{\frac{1}{2} P+\eta P^{\prime}}
$$


Differentiating (5.25) with respect to $\eta$, we get

$$
\begin{aligned}
Q^{\prime}=\left[\left(\frac{1}{2} \eta^{2} P^{2}-\alpha g^{1 / 2} \eta^{3 / 2} P+g \eta\right) P^{\prime \prime}\right. & \\
& +\frac{\left.\left(\eta P^{2}-\frac{3}{2} \alpha g^{1 / 2} \eta^{1 / 2} P+\frac{3}{2} g\right) P^{\prime}+\eta^{2} P P^{\prime 2}+\eta^{3} P^{\prime 3}\right]}{\left(\frac{1}{2} P+\eta P^{\prime}\right)^{2}} .
\end{aligned}
$$

Using the above relation and (5.25), (5.24) yields

$$
\begin{aligned}
\left(\eta^{3} P^{2}-2 \alpha g^{1 / 2} \eta^{5 / 2} P+2 g \eta^{2}\right) P^{\prime \prime} & +\left(2 g \eta+2 \eta^{2} P^{2}-4 \alpha g^{1 / 2} \eta^{3 / 2} P\right) P^{\prime} \\
& +\left(\eta^{3} P-2 \alpha g^{1 / 2} \eta^{5 / 2}\right) P^{\prime 2}-\frac{1}{2} g P=0 .
\end{aligned}
$$

For a strict similarity form of the solution, it is necessary to choose $f=0$ in (5.18). Introducing

$$
U=u /(g h)^{1 / 2}=g^{-1 / 2} \eta^{1 / 2} P(\eta)
$$

in (5.26) we get

$$
\left(U^{2}-2 \alpha U+2\right) \frac{d^{2} U}{d \eta^{2}}+(U-2 \alpha)\left(\frac{d U}{d \eta}\right)^{2}=0,
$$

which, by an interchange of the dependent and independent variables, takes the form

$$
\left(U^{2}-2 \alpha U+2\right) \frac{d^{2} \eta}{d U^{2}}+(2 \alpha-U) \frac{d \eta}{d U}=0 .
$$

Equation (5.29) is the same as (4.6). As for (4.6), the solution of (5.29) can be written as

$$
\eta=A_{1}+B_{2} \beta\left(\frac{4-\lambda_{1}^{2}}{2-\lambda_{1}^{2}}, \frac{2-2 \lambda_{1}^{2}}{2-\lambda_{1}^{2}}, \frac{U-\lambda_{1}}{2 \lambda_{1}^{-1}-\lambda_{1}}\right),
$$

where $\lambda_{1}$ is the smaller root of $\lambda^{2}-2 \alpha \lambda+2=0$. Using (5.27), (5.25) can be written as

$$
Q=\frac{g^{1 / 2} \eta(U-2 \alpha) U^{\prime}-\frac{1}{2} g^{1 / 2}\left(U^{2}-2 \alpha U+2\right)}{\eta^{1 / 2} U^{\prime}} .
$$

The boundary condition (2.11) requires $\eta^{1 / 2} Q=0$ on $\eta=0$, that is

$$
U=\lambda_{1} \text { at } \eta=0 .
$$

The other boundary condition (2.12) at $y=h$ in the light of (5.31) leads to

$$
U=\lambda_{2} \text { at } \eta=1 \text {. }
$$

Using (5.32) and (5.33) in (5.30), we finally get the solution

$$
\eta=\beta\left(\frac{4-\lambda_{1}^{2}}{2-\lambda_{1}^{2}}, \frac{2-2 \lambda_{1}^{2}}{2-\lambda_{1}^{2}}, \frac{U-\lambda_{1}}{2 \lambda_{1}^{-1}-\lambda_{1}}\right) / \beta\left(\frac{4-\lambda_{1}^{2}}{2-\lambda_{1}^{2}}, \frac{2-2 \lambda_{1}^{2}}{2-\lambda_{1}^{2}}, 1\right),
$$

where $\left(6-2 \lambda_{1}^{2}\right) /\left(2-\lambda_{1}^{2}\right)$ should not be a negative integer or zero. In Freeman's notation, $\lambda_{1}=(a)^{-1}$. 
It is of some interest to compare the above derivation with Freeman's [1]. Equations (2.9)-(2.10) can be combined to yield a first "integral"

$$
w_{1}=g(u-c) \int_{0}^{y} \frac{d y}{(u-c)^{2}} \equiv(u-c) I .
$$

Eliminating $w_{1}$ from (2.9), (2.10), and (5.35), we get an equation for $I$,

$$
\frac{\partial^{2} I}{\partial y \partial h}+I \frac{\partial^{2} I}{\partial y^{2}}=2 \frac{\partial I}{\partial y}\left[\frac{\partial I}{\partial y}-\frac{c^{\prime}(h)}{g^{1 / 2}}\left(\frac{\partial I}{\partial y}\right)^{1 / 2}\right],
$$

satisfying the boundary conditions

$$
I(h, h)=1, \quad I(h, 0)=0 .
$$

Introducing $\eta=y / h$ in (5.36), we have the PDE

$$
h \frac{\partial^{2} I}{\partial \eta \partial h}+(I-\eta) \frac{\partial^{2} I}{\partial \eta^{2}}=\frac{\partial I}{\partial \eta}+\frac{\partial I}{\partial \eta}\left[\frac{\partial I}{\partial \eta}-\frac{c^{\prime}(h) h^{1 / 2}}{g^{1 / 2}}\left(\frac{\partial I}{\partial \eta}\right)^{1 / 2}\right],
$$

with boundary conditions

$$
I(h, 1)=1, I(h, 0)=0 .
$$

Freeman sought similarity solutions of $(5.37)$ in the form $I=I(\eta)$. This comes about if $c^{\prime}(h) h^{1 / 2} / g^{1 / 2}=\alpha$, a constant. The first term in (5.37) drops out.

He solved the resulting ODE through several ingenious transformations: $J=I-\eta$, $N^{2}=J^{\prime}+1=d I / d \eta$, expressing finally $\eta$ as an incomplete $\beta$ function in $U$. Our approach is more direct. The function $U$ is governed directly by Eq. (5.28). This equation is first exactly linearized by interchanging the role of dependent and independent variables. We thus obtain a hypergeometric equation with an incomplete beta function as its solution.

6. Results and conclusions. In this paper, we have been able to generate a new class of solutions of nonlinear shallow water equations, describing sheared flow between a horizontal bottom and a free surface. The analysis carried out here vindicates the usefulness of the method of infinitesimal transformations in bringing out the hidden symmetries of the system of nonlinear PDE's and the identification of exact similarity solutions, which would otherwise be difficult to guess. The rather unusual free surface conditions pose special difficulties in the analysis of such problems. The appearance of incomplete $\beta$-function form of solutions describing free surface flows without critical level is noteworthy.

A typical profile of the horizontal velocity both for (4.12) and (5.34) is shown in Fig. 1. Both the functions (4.12) and (5.34) have the same graph. The self-propagating solution (5.34) as well as the explicitly time-dependent solution (4.12) represent what Barenblatt and Zeldovich [10] term as intermediate asymptotics, "representing behaviour of solution of wider classes of initial, boundary, and mixed problems, i.e., they describe the behaviour of these solutions away from the boundaries of the region of independent variables or, alternatively, in the region where in a sense the solution is no longer dependent on the 
details of the initial and/or boundary conditions but the system is still far from being in a state of equilibrium". It does not seem possible to show analytically that the present solutions do indeed represent intermediate asymptotics. One may have to solve the initial boundary value problem numerically, as posed by Freeman [6], for example, to arrive at this conclusion.

It is of some interest to compare the nature of free surface of the explicitly time-dependent solution,

$$
h=\frac{k\left[x+A+\frac{a_{0}}{c}\left(t+\frac{B}{l+1}\right)\right]^{2}}{(t+B)^{2}},
$$

with that obtained by other investigators for related problems. For example, Longuet-Higgins [11] has found self-similar time-dependent flows with a parabolic free surface

$$
y^{2}=\frac{3 P}{t^{3}}\left(x+\frac{P}{4 t^{3}}-Q t\right),
$$

where $P$ and $Q$ are constants. The decaying solutions (3.61) and (6.1) are quite similar. However, the distinction between the two physical problems should be clearly recognized. Longuet-Higgins' solutions describe free surface flows in space without gravitational effects and have no horizontal bottom to support. They are obtained by solving the Laplace equation in the velocity potential with Bernoulli's equation connecting the pressure and the velocity potential and the free surface being determined by the condition $p=0$. The solutions with (3.61) and (6.1) are local in nature, as they stand, since $h$ is infinitely large far away.

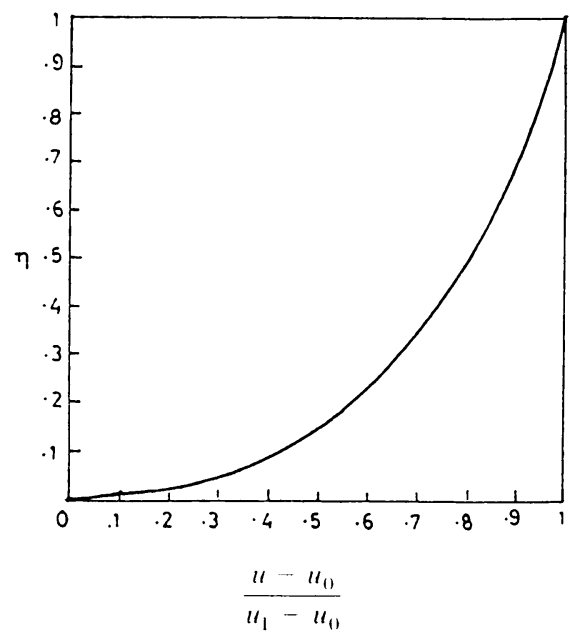

FIG. 1. A typical velocity profile with $\alpha=1.9$. 
We may also compare our solutions with linear (mostly unstable) solutions found by Varley et al. [12], which solve essentially the same problem as discussed in the present paper. For example the linear solution with the free surface

$$
\begin{aligned}
& H(x, t)=H_{0}+h_{0} \cos (2 \bar{\alpha} \bar{t}) \exp \left[\bar{t}^{2}-\bar{\alpha}^{2}\right], \\
& \bar{\alpha}=\frac{x-C_{01} t}{\lambda}, \quad \bar{t}=\frac{C_{02} t}{\lambda},
\end{aligned}
$$

with $C_{01}, C_{02}, \lambda, H_{0}$, and $h_{0}$ all constant, has a finite amount of energy at $t=0$ and takes an infinite time to become unbounded at all $x$. However, most of the nonlinear study by Varley et al. relates to flows with critical levels and does not bear comparison with the present work.

Acknowledgment. The authors wish to thank the referee for useful comments and suggestions.

\section{REFERENCES}

[1] N. C. Freeman, Simple waves on shear flows: Similarity solutions, J. Fluid Mech. 56, 257-263 (1972)

[2] P. L. Sachdev, Exact self-similar time-dependent free surface flows under gravity, J. Fluid Mech. 96, 797-802 (1980)

[3] P. L. Sachdev and A. V. Reddy, Some exact solutions describing unsteady plane gas flows with shocks, Quart. Appl. Math. XL, 249-272 (1982)

[4] P. L. Sachdev and M. C. Singh, Exact time-dependent flows for non-shallow water equations, J. Appl. Math. and Phys. (ZAMP) 35, 585-591 (1984)

[5] P. A. Blythe, Y. Kazakia and E. Varley, The interaction of large amplitude shallow-water nates with an ambient shear flon: Noncritical flon's, J. Fluid Mech. 56, 241-255 (1972)

[6] L. V. Ovsjannikov, Group properties of differential equations, translation by G. W. Bluman of Gruppovye Svoysta Differentsialny Uraveni Novosibirsk, U.S.S.R., 1962

[7] (;. W. Bluman and J. D. Cole, Similarity methods for differential equations, Springer-Verlag, New York, 1974

[8] J. D. Logan and J. D. J. Perez, Similarity solutions for reactive shock hydrodynamics, SIAM J. Appl. Math. 39. 512-527 (1980)

[9] Y. L. Luke, Special functions and their approximations, vol. 1, 2 Academic Press, New York, 1969

[10] (i. I. Barenblatt and Ya. B. Zeldovich. Self-similar solutions as intermediate asymptotics, Annual Review of Fluid Mechanics 4, 285-312 (1972)

[11] M. S. Longuet-Higgins, Self-similar, time-dependent flows with a free surface. J. Fluid Mech. 73, 603-620 (1976)

[12] E. Varley, J. Y. Kazakia and P. A. Blythe, The interaction of large amplitude harotropic waves with an ambient shear flow: Critical flows, Phil. Trans. Roy. Soc. 287, 189-236 (1977) 